Artículo Original. Enero-Abril 2017; 7(1):36-43. Recibido: 09/01/2017 Aceptado: 09/03/2017.

http://dx.doi.org/10.21929/abavet2017.71.3

\title{
Evaluación del efecto antibacterial del aceite de oliva ozonizado contra Listeria monocytogenes
}

Evaluation of the antibacterial effect of ozonized olive oil against Listeria

monocytogenes

\section{Godínez-Oviedo Angélica*1 angelica_godinez@uahe.edu.mx, Zamora-Rodríguez Zullyt ${ }^{2}$ efleitas@infomed.sld.cu, Martínez-Juárez Víctor ${ }^{1}$ victormj@uaeh.edu.mx , Fleitas- González Eduardo ${ }^{3}$ efleitas@infomed.sld.cu, Hernández-Rosado Abigail adrianhg10@hotmail.com Peña-Jiménez Francisco**1 fjpenj@hotmail.com}

1 Universidad Autónoma del Estado de Hidalgo. Instituto de Ciencias Agropecuarias. Tulancingo de Bravo, Hidalgo. Mexico. 'Departamento de Farmacología, Unidad de Sustancias Ozonizadas y Productos Naturales, Centro Nacional de Investigaciones Científicas de Cuba. ${ }^{3}$ Clínico Privado. Director de la Clínica Veterinaria "Almiqui", La Habana, Cuba. *Autor responsable. ${ }^{*}$ Autor de correspondencia: Peña-Jiménez Francisco. Universidad Autónoma del Estado de Hidalgo. Instituto de Ciencias Agropecuarias. Rancho Universitario. Av. Universidad Km. 1. Ex Hacienda Aquetzalpa, Apartado Postal No. 32, Tulancingo de Bravo, Hidalgo.

\section{RESUMEN}

Los aceites vegetales ozonizados debido a su capacidad antimicrobiana, son una nueva alternativa para ser empleados en humanos y animales contra microorganismos patógenos. El objetivo de este estudio fue determinar el efecto antibacterial del aceite de oliva ozonizado contra Listeria monocytogenes. Tres diferentes aceites ozonizados fueron empleados: T1: aceite de oliva ozonizado y almacenado por seis meses a $4^{\circ} \mathrm{C}$; T2: aceite de oliva ozonizado elaborado al momento del análisis y un aceite comercial (Oleozon $\AA^{\circledR}$ ). Se determinó el índice de peróxido del aceite T2 y Oleozon ®. La capacidad antibacterial se evaluó mediante la técnica de difusión en pozo empleando diferentes concentraciones del patógeno. Los índices de peróxidos se encontraron entre un rango de $400-490 \mathrm{mmol} / \mathrm{kg}$ y $500-800 \mathrm{mmol} / \mathrm{kg}$ para T2 y Oleozon ${ }^{\circledR}$, respectivamente. Los aceites ozonizados mostraron efecto antimicrobiano, observando que a menor concentración del inoculo mayor efecto. El efecto antimicrobiano del aceite comercial tuvo una diferencia estadísticamente significativa $(p<0.05)$ respecto a los otros aceites, teniendo un mayor efecto. Por otra parte, no se observaron diferencias entre los aceites T1 y T2, por lo cual el almacenamiento a estas condiciones no afecta el efecto antimicrobiano. Los aceites vegetales ozonizados tienen una alta capacidad antibacterial contra L. monocytogenes

Palabras clave: Aceite de oliva ozonizado, antimicrobiano, Listeria monocytogenes.

\begin{abstract}
The vegetable ozonized oils are a new alternative to treat humans and animals against pathogens, due to their antimicrobial capacity. The aim of this study was to determine the antimicrobial effect of olive ozonized oil against Listeria monocytogenes. Three different ozonized oils were used: T1: olive ozonized oil stored for 6 months at $4^{\circ} \mathrm{C}, \mathrm{T} 2$ : olive ozonized oil produced in the moment of the test, and a commercial ozonized oil (Oleozon $\AA$ ). The peroxide index of the oils was determined using a volumetric method. Also, the antimicrobial activity was evaluated using a diffusion method with different concentrations of the inocula. The peroxide index was in a range of 400 to $490 \mathrm{mmol} / \mathrm{kg}$ and 500 to $800 \mathrm{mmol} / \mathrm{kg}$ for the T2 and Oleozon® oils, respectively. All the ozonized oils showed antimicrobial effect, finding that the higher activity was presented with a lower concentration of the inocula. The commercial oil has a significant difference $(p<0.05)$
\end{abstract}


respect to the T1 and T2 oils, having a higher effect. On the other hand, the T1 and T2 treatments did not show significant differences between them, whence the storage at this conditions did not affect the antimicrobial effect.

Keywords: ozonized olive oil, antimicrobial, Listeria monocytogenes.

\section{INTRODUCCIÓN}

El ozono $\left(\mathrm{O}_{3}\right)$ es una molécula compuesta por tres átomos de oxígeno, la cual posee actividad antibacteriana, antiviral y antifúngica; dicha actividad ha sido altamente estudiada desde hace años. De la misma forma se ha evaluado la actividad antimicrobiana de productos que sufren un proceso de oxidación frente a este compuesto, como son el agua ozonizada y los aceites vegetales ozonizados.

Los aceites vegetales ozonizados, se obtienen después de la oxidación generada por el ozono a los ácidos grasos y otras sustancias presentes en los aceites vegetales (Martínez-Sánchez et al., 2012). Durante la reacción de ozonización de los aceites vegetales se producen lipoperóxidos, hidroperóxidos, peróxidos, ozónidos, aldehídos y cetonas (Díaz et al., 2005; Martínez-Sánchez et al., 2012).

Los ácidos grasos poli-insaturados son los más susceptibles a la oxidación durante el proceso (Díaz et al., 2005). El rendimiento de los productos de la ozonización depende de las condiciones de la reacción, como son: la temperatura, el generador de ozono, la concentración de ozono empleada y el tiempo de reacción (Almeida et al., 2013). Los aceites de oliva, sésamo, maní, coco, teobroma, soja y jojoba, son algunas de las materias primas que se han empleado para la realización de estos productos (Meléndez et al., 2008). De la misma forma, en el mercado ya existe diversos productos como es el caso del Oleozon $\AA^{\circledR}$ desarrollado en Cuba a base de aceite de girasol; el Cocozone $\AA$ elaborado en Inglaterra con aceite de coco; el $\mathrm{OOO} \otimes$ producto canadiense elaborado con aceite de oliva y el O2-ZAP® producto estadunidense a base de aceite de oliva (Skalska et al., 2009).

La importancia de los aceites ozonizados radica en que los compuestos presentes en los mismos, pueden actuar como germicidas, agentes de restauración de tejido e inmuneestimulantes (Martínez-Sánchez et al., 2012). Debido a las propiedades de los aceites vegetales ozonizados, estos pueden ser una nueva alternativa para combatir microorganismos patógenos y deterioradores; ya que hoy en día, debido al uso irracional y exagerado de los antibióticos, diversos microorganismos han generado multiresistencia a los mismos; por lo cual es necesario emplear nuevas alternativas, como son los agentes antimicrobianos de origen vegetal. Algunas de las ventajas de este producto son: menor costo respecto a otros antibióticos, no requieren estabilizantes, son de origen natural y que tienen un efecto similar o superior al de antibióticos presentes en el mercado (Sifontes et al., 2015). 
El efecto antimicrobiano de los aceites ozonizados se ha atribuido a los ozónidos, peróxidos, compuestos derivados del proceso como el formaldehido y al ozono libre, presente en los aceites (Menéndez et al., 2008; Guinesi, 2011; lorio et al., 2015). Sin embargo, durante el almacenamiento los aceites ozonizados sufren reacciones de despolimerización, por lo cual esto puede modificar sus propiedades químicas, físicas y microbiológicas (Martínez-Sánchez et al., 2012). Por lo anterior es necesaria la realización de estudios para establecer la vida útil de estos productos y sus propiedades contra microorganismos específicos, para poder definir su aplicación y uso como medicina alternativa para humanos y animales contra diversas patologías.

Por lo cual, el objetivo de este estudio fue evaluar el poder antibacteriano del aceite de oliva ozonizado contra Listeria monocytogenes.

\section{MATERIAL Y MÉTODOS}

\section{Ozonización del aceite de oliva}

Se empleó un aceite de oliva extra virgen comercial, el cuál de acuerdo a lo reportado en su etiqueta contiene $78.5 \%$ de grasas monoinsaturados (omega 9 ), $7.1 \%$ de grasas poli instaurados (Omega 3 y 6 ) y $14 \%$ de grasas saturadas; $100 \mathrm{ml}$ del aceite de oliva. Se ozonizaron empleado un ozonizador de aceite de dos potencias (OzonoBio3, Modelo AO5), burbujeándolo por una hora a una concentración de ozono de $50 \mathrm{mg} / \mathrm{ml}$. El ozonizador estaba conectado a un generador de oxígeno (Respironics Everflo, Philips) generando un flujo de oxígeno de $2 \mathrm{~L} / \mathrm{min}$.

Para el estudio se empelaron dos aceites de oliva ozonizados, uno después de 6 meses de almacenamiento a $4^{\circ} \mathrm{C}(\mathrm{T} 1)$ y otro realizado al momento de realizar el experimento (T2); además se empleó un aceite de girasol ozonizado comercial (OLEOZON®)

\section{Determinación del índice de peróxidos}

El índice de peróxido se determinó empleado la tecina reportada por Farmacopedia Británica (British Parmacopeia, 2000); se evaluó el índice de peróxido para los aceites de oliva ozonizado T2 y Oleozon®. Para su realización se colocaron $5 \mathrm{~g}$ de la muestra en un matraz con $30 \mathrm{ml}$ de una solución ácido acético-cloroformo 2:3. La muestra se agitó hasta disolverse totalmente y se le adicionó $0.5 \mathrm{ml}$ de una solución saturada de yoduro de potasio y se dejó reposar por un minuto. Al finalizar el tiempo se le adicionaron $30 \mathrm{ml}$ de agua destilada y procedió a titularse con tiosulfato de sodio $0.01 \mathrm{~N}$, hasta obtener una coloración ligeramente amarilla. Después de obtener dicha coloración, se le adicionó 0.5 $\mathrm{ml}$ de una solución indicadora de almidón, y se continuó titulando hasta la aparición de un color azul. Bajo las mismas condiciones se realizó la titulación de un blanco. El índice de peróxido se expresó en mini equivalentes de peróxido en un kg de aceite y se calculó empleando la siguiente formula. 
I.P = Índice de Peróxido

$$
I . P=\frac{\left(A-A_{1}\right) * N * 1000}{M}
$$

$A=$ Mililitros de solución de tiosulfato de sodio gastados en la titulación de la muestra. $A 1=m l$ de solución de tiosulfato de sodio gastados en la titulación del blanco.

$\mathrm{N}=$ Normalidad de la solución de tiosulfato de sodio.

$\mathrm{M}=$ Masa de la muestra en gramos

\section{Cepas utilizadas}

La cepa bacteriana que se empleó fue Listeria monocytogenes (ATCC 19115).

\section{Preparación del inoculo}

La cepa de L. monocytogenes se activó transfiriendo $40 \mu \mathrm{L}$ del cultivo, mantenido en congelación a un tubo con $3 \mathrm{ml}$ de caldo soya tripticaseina (CST, Dibico; México) y se incubó durante $24 \mathrm{~h}$ a $35^{\circ} \mathrm{C}$. Posteriormente se estrió en agar soya tripticaseina (Dibico; México) con $0.6 \%$ de extracto de levadura (Bioxon; México) (ASTEL) y se incubó a las mismas condiciones que el paso anterior. Después de las $24 \mathrm{~h}$, se tomó una azada de la cepa estriada en la caja y se ajustó con solución salina isotónica (SSI), hasta obtener una turbidez equivalente al estándar 3 de McFarland ( 9 X108 UFC/ml).

Para las pruebas microbiológicas se empleó la suspensión de la bacteria sin diluir, la segunda dilución y la cuarta dilución. Las diluciones decimales fueron realizadas con SSI.

\section{Determinación del efecto antibacteriano por el método de difusión en pozo}

El efecto antibacteriano se determinó extendiendo en placas de ASTEL $100 \mu \mathrm{L}$ de las suspensiones bacterianas ( $9 \times 10^{8} \mathrm{UFC} / \mathrm{mL}, 9 \times 10^{6} \mathrm{UFC} / \mathrm{mL}, 9 \times 10^{4} \mathrm{UFC} / \mathrm{mL}$ ). Posteriormente se dejó secar la caja durante $15 \mathrm{~min}$ y se realizaron 4 pozos $(8 \mathrm{~mm}$ de diámetro) por caja; en cada pozo se colocaron $80 \mu \mathrm{L}(\sim 70 \mathrm{mg})$ de los aceites ozonizados: 1) control negativo: aceite de oliva sin ozonizar; 2) T1: aceite de oliva ozonizado almacenado 6 meses a $4^{\circ} \mathrm{C}$; 3) T2: aceite de oliva ozonizado al momento y 4) T3: Oleozon® (control positivo). Las cajas se incubaron $24 \mathrm{~h}$ a $35^{\circ} \mathrm{C}$. Transcurrido el tiempo de incubación se midieron los halos de inhibición. El experimento se realizó por cuadriplicado.

Se realizó una ANOVA para evaluar estadísticamente del efecto antimicrobiano, y la prueba Tukey para la comparación de medias; empleando el programa JMP 8.0.

\section{RESULTADOS Y DISCUSIÓN}

El índice de peróxido de los aceites ozonizados, se encontró en un rango de 400-490 $\mathrm{mmol} / \mathrm{kg}$ y $500-800 \mathrm{mmol} / \mathrm{kg}$ para el aceite de oliva ozonizado y para el Oleozon $\AA$, respectivamente. 
El análisis ANOVA del efecto antimicrobiano, mostró que tanto la concentración del inoculo $(p<0.0001)$, como el tratamiento empleado $(p<0.0001)$, tuvo un efecto significativo; obteniendo que ha menor concentración del inoculo, se obtuvo mayores halos de inhibición; y que en todos los casos los aceites ozonizados de oliva, tuvieron una diferencia estadísticamente significativa $(p<0.05)$ respecto al aceite de girasol ozonizado comercial (Oleozon $\AA$ ), siendo mayor el efecto de este último. La diferencia entre los aceites ozonizados de oliva y el de marca comercial, puede deberse tanto a la materia prima, como al índice de peróxido.

La composición de los ácidos grasos saturados e insaturados de los aceites vegetales, es diferente dependiendo su origen; por lo cual los lipoperóxidos que se generan durante la ozonización, también varían (Díaz et al., 2001).

El aceite comercial está hecho a base de girasol, y los aceites T1 y T2 a base de aceite de oliva. De la misma forma se puede observar que el Oleozon $\AA^{\circledR}$ tiene mayor índice de peróxido, lo que puede influir en su actividad antimicrobiana. Díaz et al., (2006) observaron el mismo comportamiento al probar aceite de girasol ozonizado con diferentes índices de peróxidos, contra Staphylococcus aureus, Pseudomonas aeruginosa, Bacillus subtilis y Escherichia coli; encontrando que a mayor índice de peróxido, existía una mayor actividad antimicrobiana. Por lo cual sería necesario evaluar el aumento del índice de peróxido conforme al tiempo, y determinar el punto de saturación, con la finalidad de poder obtener un aceite ozonizado con mayor actividad antibacterial. Se ha observado que el incremento de 1 a $3 \mathrm{~h}$ de ozonización duplica el índice de peróxido (Fernández et al., 2006).

En la Tabla 1 se observan los halos de inhibición $(\mathrm{mm})$ de los diferentes aceites ozonizados a diferentes concentraciones bacterianas. Se observa que ambos aceites de oliva ozonizados no tuvieron diferencias estadísticamente significativas entre sí, lo que indica que el almacenamiento por 6 meses a $4^{\circ} \mathrm{C}$ no afecta la actividad antimicrobiana del aceite ozonizado. Similar a esto, se ha encontrado que el Oleozon $\AA$ tiene una estabilidad de 6 meses a temperatura ambiente, y más de un año a temperatura de $-10^{\circ} \mathrm{C}$ a $8^{\circ} \mathrm{C}$; después de ese periodo disminuye la actividad antimicrobiana (Sechi et al., 2001). Entre mayor es la temperatura de almacenamiento conforme pasa el tiempo, existe una disminución en el índice de peróxidos, y un aumento en el índice de acidez; pero a temperaturas de $-20^{\circ} \mathrm{C}$ a $4^{\circ} \mathrm{C}$ no se observan cambios significativos en estos parámetros durante un año (Moureun et al., 2015). 


\begin{tabular}{llll}
\hline Tratamiento & \multicolumn{2}{c}{ Diámetro de inhibición $(\mathrm{mm})$} \\
& Inoculo: $9 \times 10^{7} \mathrm{UFC} / \mathrm{mL}$ & Inoculo: $9 \times 10^{5} \mathrm{UFC} / \mathrm{mL}$ & Inoculo: $9 \times 10^{3} \mathrm{UFC} / \mathrm{mL}$ \\
\hline Aceite de oliva ozonizado (T1) & $17.5 \pm 1.3^{\mathrm{b}}$ & $26.4 \pm 3^{\mathrm{b}}$ & $34 \pm 0.8^{\mathrm{b}}$ \\
Aceite de oliva ozonizado (T2) & $18.3 \pm 2.1^{\mathrm{b}}$ & $24 \pm 1.6^{\mathrm{b}}$ & $32.25 \pm 0.5^{\mathrm{b}}$ \\
Oleozon $\AA$ & $30 \pm 0.8^{\mathrm{a}}$ & $44.8 \pm 3.2^{\mathrm{a}}$ & $50 \pm 1.4^{\mathrm{a}}$ \\
\hline a, b Cada valor representa el promedio de cuatro replicas \pm la desviación estándar. Las letras diferentes expresan \\
diferencia significativa con la prueba Tukey a 0.05
\end{tabular}

Tabla 1. Efecto antibacterial de los aceites vegetales ozonizados contra L. monocytogenes

Los ozónidos son uno de los compuestos que se han relacionado con la actividad antimicrobiana de los aceites ozonizados, aunque no son los únicos responsables (Díaz et al., 2001). Estos compuestos durante el almacenamiento y el aumento de la temperatura tienden a descomponerse y formar ácido nonanoico y ácido azelaico; dichos compuestos también tienen efecto antimicrobiano (Moureun et al., 2015); esta puede ser la razón por la cual el efecto antimicrobiano no disminuye. Siendo importante analizar sí los ozónidos o los ácidos tienen mayor efecto, con la finalidad de estandarizar la vida útil y óptima de los aceites ozonizados.

Se han realizado estudios similares contra otros microorganismos, en los cuales se han encontrado diámetros de inhibición superiores (Montrvecchi et al., 2013) e inferiores (lorio et al., 2015); sin embargo no es posible realizar comparaciones, debido a que los microorganismos empleados no son los mismos, a que en algunos estudios evalúan la cantidad de ozono libre y en otros el índice de peróxidos, y a la concentración y viabilidad del inoculo.

En la Figura I se observan los halos de inhibición dependiendo de la concentración.

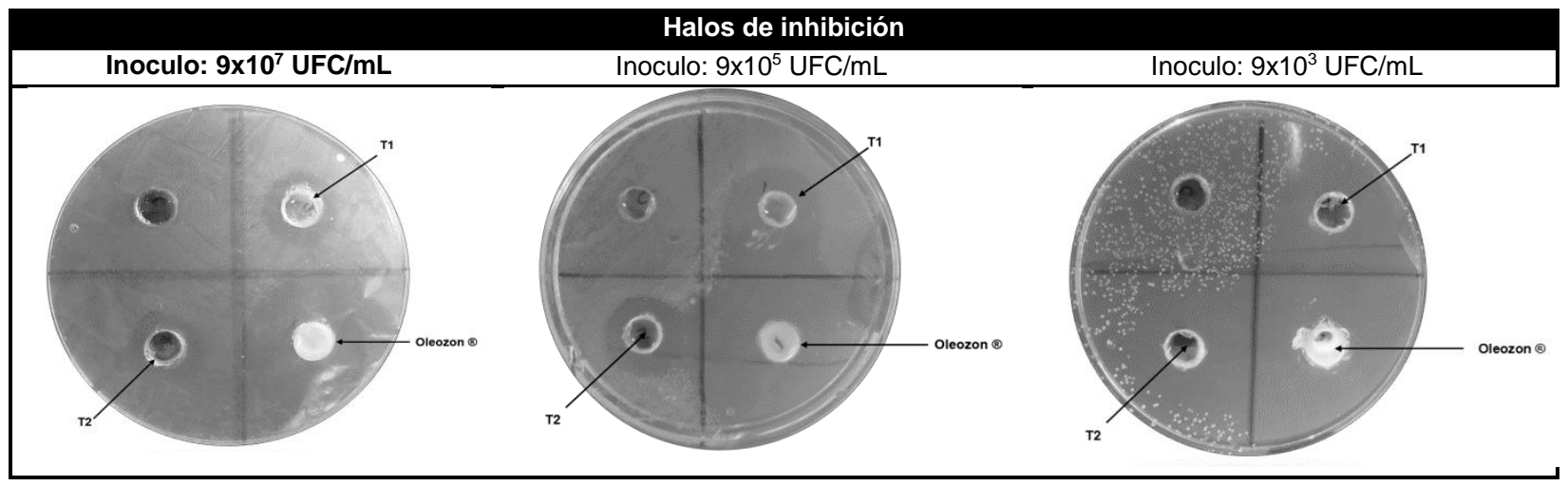

Figura I. Efecto antimicrobiano de los aceites ozonizados contra Listeria monocytogenes. 


\section{CONCLUSIÓN}

El aceite de oliva ozonizado mostró un buen efecto antibacteriano contra $L$. monocytogenes, en todas las diferentes concentraciones de patógeno, a pesar de que a mayor concentración del inoculo se observaron menores halos de inhibición. De la misma forma se determinó que el almacenamiento por 6 meses a temperatura de $4^{\circ} \mathrm{C}$ del aceite de oliva ozonizado, no afectó su actividad antimicrobiana.

Es necesaria la realización de más estudios, con la finalidad de determinar la vida útil de estos aceites y en base a ellos poder emplearlos en diversas áreas.

\section{AGRADECIMIENTOS}

Los autores de este artículo agradecen a la Universidad Autónoma del Estado de Hidalgo y al Programa para el Desarrollo Profesional Docente, para el Tipo Superior (PRODEP) por el apoyo facilitado para la realización de este trabajo

\section{LITERATURA CITADA}

ALMEIDA NR, Beatriz A, Micheletti AC, Arruda EJ. 2013. Ozonized vegetable oils and therapeutic properties: A review. Orbital-The Electronic Journal of Chemistry. 4(4):31326. ISSN 1984-6428, DOI: 10.17807/orbital.v4i4.467

BRITISH Pharmacopoeia. 2000. Appendix XF, IA, IB. Peroxide value. http://www.uspbpep.com/bp2008/data/899.asp

DÍAZ M, Lezcano I, Molerio J, Hernández F. 2001. Spectroscopic characterization of ozonides with biological activity. Ozone Science and Engineering. 23(1):35-40. ISSN: 1547-6545.DOI: 10.1080/01919510108961986

DíAZ MF, Gavín JA, Gómez M, Curtielles V, Hernández F. 2006. Study of ozonated sunflower oil using $1 \mathrm{H} \mathrm{NMR} \mathrm{and} \mathrm{microbiological} \mathrm{analysis.} \mathrm{Ozone:} \mathrm{Science} \mathrm{and}$ Engineering. 28(1):59-63. ISSN: 1547-6545. DOI: 10.1080/01919510500479239

DÍAZ MF, Gavín Sazatornil JA, Ledea O, Hernández F, Alaiz M, Garcés R. 2005. Spectroscopic characterization of ozonated sunflower oil. Ozone: Science and Engineering. 27(3):247-53. ISSN: 1547-6545. DOI: 10.1080/01919510590945822

FERNÁNDEZ TI, Curtiellas Piñol V, Sánchez Urrutia E, Gómez Regueiferos M. 2006. In vitro antimicrobial activity of ozonized theobroma oil against Candida albicans. Ozone: Science and Engineering. 28(3):187-90. ISSN: 1547-6545. DOI: $10.1080 / 01919510600689380$ 
IORIO FB, Liberatore AM, Koh IH, Otani C, Camilo FF. 2016. Ozonated Mineral Oil: Preparation, Characterization and Evaluation of the Microbicidal Activity. Ozone: Science and Engineering. 38(4):253-60. ISSN: 1547-6545. DOI: 10.1080/01919512.2015.1128801

KIM HS, Noh SU, Han YW, Kim KM, Kang H, Kim HO, Park YM. 2009. Therapeutic effects of topical application of ozone on acute cutaneous wound healing. Journal of Korean Medical Science. 24(3):368-74. ISSN: 1011-8934 DOI: 10.3346/jkms.2009.24.3.368

MARTÍNEZ-SÁNCHEZ G, Re L, Davison GP, Delaporte RH. 2012. Las aplicaciones médicas de los aceites ozonizados: actualización. Revista Española de Ozonoterapia. 2(1):121-39. ISSN: 2174-3215

MONTEVECCHi M, Dorigo A, Cricca M, Checchi L. 2013. Comparison of the antibacterial activity of an ozonated oil with chlorhexidine digluconate and povidone-iodine. A disk diffusion test. New Microbiologica. 36: 289-302. ISSN: 1121-7138

MOUREU S, Violleau F, Ali Haimoud-Lekhal D, Calmon A. 2016. Influence of Storage Temperature on the Composition and the Antibacterial Activity of Ozonized Sunflower Oil. Ozone: Science and Engineering. 38(2):143-149. ISSN: 1547-6545. DOI: 10.1080/01919512.2015.1128319

RAJABI O, Sazgarnia A, Abbasi F, Layegh P. 2015. The activity of ozonated olive oil against Leishmania major promastigotes. Iranian Journal of Basic Medical Sciences. 18(9):915. ISSN: 2008-3874. DOI: 10.22038/IJBMS.2015.5215

SECHI LA, Lezcano I, Nunez N, Espim M, Duprè I, Pinna A, Molicotti P, Fadda G, Zanetti S. 2001. Antibacterial activity of ozonized sunflower oil (Oleozon). Journal of Applied Microbiology. 90(2):279-284. ISSN: 1365-2672. DOI: 10.1046/j.1365-2672.2001.01235.x

SIFONTES AB, Marcos R, Avila EE, Villalobos-Duno HL. 2015. Uso clínico de los aceites ozonizados y su amplio espectro de las aplicaciones. Botica. 35:1-5. ISSN: 2443-4388

SKALSKA K, Ledakowicz S, Perkowski J, Sencio B. 2009. Germicidal properties of ozonated sunflower oil. Ozone: Science and Engineering. 31(3):232-237. ISSN: 15476545. DOI: $10.1080 / 01919510902838669$

TRAVAGLI V, Zanardi I, Bocci V. 2009. Topical applications of ozone and ozonated oils as anti-infective agents: an insight into the patent claims. Recent Patents on Anti-infective Drug Discovery. 4(2):130-142. ISSN: 2212-4071. DOI: 10.2174/157489109788490271 